\title{
Pacientes con trastorno por uso de alcohol: resultados iniciales de un registro multicéntrico en la Red de Trastornos Adictivos-RTA. Estudio CohRTA
}

\author{
Patients with alcohol use disorder: initial results \\ from a prospective multicenter registry in the Spanish \\ Network on Addiction Disorders. CohRTA Study
}

\begin{abstract}
Arantza Sanvisens*, Paola Zuluaga* (ambas autoras participan por igual), Inmaculada Rivas**, Gabriel Rubio***, Antoni Gual ${ }^{* * * *}$, Marta Torrens*****, Antoni Short******, Francisco Javier Áldvarez $* * * * * * *$, Jordi Tor********, Magí FarRé********, Fernando Rodriguez de FonSECA $* * * * * * * * *$, Roberto MUGA $* * * * * * * *$ Y COHRTA.
\end{abstract}

\begin{abstract}
* Institut d'Investigació Germans Trias i Pujol-IGTP, Badalona; ** Centro de Atención a las Drogodependencias-Centro Delta, Institut Municipal de Serveis Personals, Badalona; *** Servicio de Psiquiatría, Hospital 12 de Octubre, Madrid. Universidad Complutense de Madrid; **** Servicio de Psiquiatría, Hospital Clínic, Barcelona, Universitat de Barcelona; ***** Instituto de Neuropsiquiatría y Adicciones, Parc de Salut Mar, Barcelona. Universitat Autònoma de Barcelona; ****** Unidad de Alcohol, Hospital Universitario Son Espases, Palma de Mallorca; ******** Servicio de Farmacología, Hospital Clínico, Valladolid, Universidad de Valladolid; ******** Servicios de Medicina Interna y Famacología Clínica, Hospital Universitari Germans Trias i Pujol, Universitat Autònoma de Barcelona, Badalona; ********* Instituto de Investigación Biomédica-IBIMA, Málaga.
\end{abstract}

\section{Resumen}

El Programa Alcohol de la Red de Trastornos Adictivos (RTA) requiere de un estudio clínico longitudinal para dar respuesta a preguntas de investigación en el trastorno por uso de alcohol. El proyecto CohRTA es un estudio multicéntrico de investigación cooperativa que se pone en marcha para mejorar la prevención secundaria y el diagnóstico precoz de los procesos patológicos asociados al trastorno por uso de alcohol.

Método: estudio observacional en cohorte multicéntrica de pacientes mayores de 18 años que solicitan tratamiento del trastorno por primera vez y autorizan su participación. La información clínica se recoge en una plataforma online diseñada para el estudio y puede ir acompañada de una muestra biológica que se deposita en un biobanco. Se recogen datos basales y prospectivos, sociodemográficos, epidemiológicos, clínicos y de tratamiento. A diciembre de 2015 son 10 los centros proveedores de pacientes y se espera reclutar más de 1.000 pacientes en los próximos años.

Resultados: se dispone de 344 pacientes ( $77 \%$ hombres) que cumplen los criterios de inclusión en el estudio y con una edad de 50 años (RIQ: 43-55 años). La edad de inicio de consumo de alcohol fue de 15 años (RIQ: 14-18 años) y un $61 \%$ tenían antecedente familiar de trastorno por uso de alcohol. Durante los 30 días previos al inicio del tratamiento los pacientes bebían 12.5 UBE/ día (RIQ: 7.1-20 UBE/día), el 72\% fumaba tabaco y el 30\% consumía cocaína. Conclusiones: Disponer de una cohorte abierta y multicéntrica de pacientes con trastorno por uso de alcohol será útil para analizar las consecuencias del abuso de alcohol, potenciar la investigación traslacional y añadir valor a la investigación clínica y básica del Programa Alcohol dentro de RTA/RETICS. Con una cohorte bien establecida y representativa se espera aumentar la cantidad y calidad científica en relación a las complicaciones del trastorno por uso de alcohol y sus consecuencias clínicas y sociales en España.

Palabras clave: Trastorno por uso de alcohol; Estudio de cohorte; Investigación.

\begin{abstract}
The Alcohol Program of the Spanish Network on Addictive Disorders-RTA requires a longitudinal study to address different research questions related to alcoholism. The cohort study (CohRTA) focuses on patients seeking treatment for alcohol use disorder, as a multicentre, collaborative research project aimed to improve secondary prevention and early diagnosis of pathological processes associated with the disorder.

Methods: multicentre cohort study in adults ( $>18$ years) seeking their first treatment of the disorder. Patients sign an informed consent and data is collected in an online platform specifically designed for the study; patients are also requested to provide biological samples that are stored in a biobank. Baseline and prospective, socio-demographic, epidemiological, clinical and treatment data are collected. Currently there are 10 participating centres that expect to recruit more than 1,000 patients.

Results: As of December 2015, 344 patients (77\% men) were included. Median age at admission was 50 years (IQR: $43-55$ years). Median age at the start of alcohol consumption was 15 years (IQR: $14-18$ years) and $61 \%$ of cases reported antecedents of alcohol use disorder in the family. During the 30 days prior to admission, alcohol consumption amounted to 12.5 SDU/day (IQR: 7.1-20 SDU/ day), $72 \%$ of the patients were tobacco smokers and $30 \%$ currently used cocaine. Organising an open cohort of patients with alcohol use disorder may be crucial to better understand the clinical consequences of alcoholism in Spain. This cohort may potentiate quantitative and qualitative research within the Spanish Network on Addictive Disorders-RTA/RETICS. Having a well-established, representative cohort of patients will increase translational research on consequences of alcoholism in our country.

Keywords: Alcohol use disorder; Cohort study; Research.
\end{abstract}

Recibido: Marzo 2016; Aceptado: Octubre 2016.

Enviar correspondencia a:

Dr. Roberto Muga. Servicio de Medicina Interna. Hospital Universitari Germans Trias i Pujol. Ctra. Canyet s/n. 08916, Badalona E-mail: rmuga.germanstrias@gencat.cat 
L a Organización Mundial de la Salud estima que 76 millones de personas tienen abuso o dependencia de alcohol en el mundo (World Health Organization, 2010). En España, la prevalencia de consumo de alcohol es elevada, ya que el $79 \%$ de la población general entre 15 y 64 años ha consumido alcohol en el último año (84\% de los hombres, $73 \%$ de las mujeres) y un $11 \%$ bebe alcohol diariamente según la encuesta domiciliaria de 2009-2010 (Observatorio Español sobre Drogas, 2011). Esta misma encuesta sitúa a los bebedores de riesgo (ingesta de alcohol superior a 50 gramos/día en hombres y 30 gramos/día en mujeres) en el $4,4 \%$ de la población general. En el grupo de edad entre 15 y 24 años, la prevalencia del consumo de alcohol de riesgo en mujeres es del 6\% y en hombres del 5\% (Observatorio Español sobre Drogas, 2011) .

Los problemas de salud relacionados con el abuso de alcohol son relativamente frecuentes en la población española y por ello en el sistema sanitario. Además, cada año cerca de 30.000 personas solicitan tratamiento del trastorno y hasta un $61 \%$ de ellas se observa que también han consumido cocaína (Observatorio Español sobre Drogas, 2011).

El trastorno por uso de alcohol es una enfermedad crónica que puede cursar con múltiples alteraciones orgánicas. El daño que el alcohol causa en órganos y tejidos depende esencialmente de la cantidad total de etanol ingerida a lo largo de la vida y del patrón de consumo, aunque también intervienen variables genéticas, biológicas y ambientales; entre las principales alteraciones orgánicas del alcoholismo, ahora definido como trastorno por uso de alcohol según el DSMV (American Psychiatric Association, 2013), destacan las hepáticas, neuro-psiquiátricas, cardiovasculares, infecciosas y el cáncer; es más, el riesgo de padecer una enfermedad asociada al abuso de alcohol empieza con niveles relativamente bajos de consumo diario de alcohol (30g/día) (Thomas et al., 2000).

El pronóstico de los múltiples trastornos orgánicos derivados del abuso de alcohol también depende de diversos factores. El alcohol puede causar lesiones en órganos y sistemas tras un consumo prolongado pero también daño a corto plazo, tras ingestas de alcohol en atracones (binge drinking) o en el contexto de intoxicaciones etílicas. Existen también marcadas diferencias según el sexo ya que las mujeres tienen mayor riesgo de desarrollar enfermedad hepática que los hombres y en los últimos años se insiste también en el mayor riesgo de cáncer de mama en bebedoras habituales (Bagnardi, Blangiardo, La Vecchia, y Corrao, 2001). Por otro lado, el consumo exagerado de alcohol explicaría una parte importante de la mortalidad por lesiones no intencionadas (accidentes, ahogamiento, hipotermia, quemaduras), intencionadas (suicidio) u otras enfermedades graves que acarrean una elevada mortalidad durante el episodio agudo (Naimi et al., 2003). En todo caso, la mortalidad de pacientes con trastorno por uso de alcohol es elevada y puede llegar a ser hasta 20 veces superior a la de la población general de la misma edad (Fuster et al., 2015; Rivas et al., 2013; Roerecke y Rehm, 2013).

Otro aspecto a tener en cuenta en la epidemiología actual del trastorno por uso de alcohol es la concurrencia del consumo de otras sustancias, en particular de cocaína, cannabis y tabaco (Fuster et al., 2015; Observatorio Español sobre Drogas, 2011; Rivas et al., 2013) El consumo de cocaína en combinación con alcohol aumenta los niveles plasmáticos de cocaína en hasta un $30 \%$ con lo que ello implica en el riesgo de de desarrollar una enfermedad cardiovascular, amén de generar un intermediario metabólico, el cocaetileno de gran potencia psicotrópica y efectos cardiotóxicos (Pennings, Leccese, y Wolff, 2002). Desde una perspectiva conductual, el consumo de cocaína facilita el consumo de alcohol ya que consumir cocaína permitiría beber alcohol durante más tiempo, lo que a su vez puede incrementar la cantidad de cocaína consumida (Gossop, Manning, y Ridge, 2006).

Es bien sabido que la información clínica que aportan las series de casos es esencial para mejorar la calidad de la asistencia y actualizar el pronóstico de cualquier enfermedad. En ese sentido, disponer de una cohorte de casos con trastorno por uso de alcohol puede resultar clave para mejorar el tratamiento de la enfermedad y potenciar la investigación centrada en el paciente. De hecho, revitalizar la investigación clínica está en el orden del día de las agencias españolas y europeas de investigación; además, la asistencia a pacientes debiera ser el puente entre ciencia básica y efectividad clínica (Pons, Rodés, Andreu, y Arenas, 2013).

La Red de Trastornos Adictivos (RTA) es una de las Redes Temáticas de Investigación Cooperativa en Salud (RETICS) del Instituto de Salud Carlos III. Desde su inicio en 2003, los objetivos científicos de la RTA se centran en investigar en el trastorno por uso de sustancias y en la etapa iniciada en 2013, en las dos sustancias con mayor impacto en nuestra sociedad: alcohol y cocaína.

Desde el Programa Alcohol de la RTA se ha impulsado la creación de una cohorte multicéntrica para el estudio de pacientes que solicitan tratamiento del trastorno por primera vez (proyecto CohRTA). En los estudios de cohorte, los pacientes se seleccionan en función de una determinada característica o exposición y una vez visitados en la primera ocasión son seguidos en el centro de reclutamiento lo que permite analizar la evolución a largo plazo y la aparición de incidencias clínicas en función de distintos factores de exposición. Los objetivos y plan de actuación de este estudio de la RTA se han presentado en foros científicos de la Sociedad Española de Medicina Interna (SEMI) y de la Sociedad Científica Española de Estudios sobre Alcohol, Alcoholismo y otras Toxicomanías (Socidrogalcohol). Además del soporte económico del ISCIII, el proyecto CohRTA 
cuenta con financiación adicional del Plan Nacional sobre Drogas (PNSD) a través de las ayudas para la investigación de 2014; la ayuda adicional del PNSD ha supuesto la ampliación del proyecto a centros clínicos no pertenecientes a la RTA, lo que permitirá aportar un mayor número de casos a la cohorte y ampliar la validez externa.

El objetivo último del estudio es establecer una plataforma estable de centros asistenciales para potenciar el conocimiento clínico y básico en el trastorno por uso de alcohol.

\section{Metodología}

\section{Diseño del estudio}

CohRTA es una cohorte abierta, prospectiva y multicéntrica de adultos que solicitan tratamiento del trastorno por uso de alcohol por primera vez. El estudio está anclado en centros asistenciales del Sistema Nacional de Salud que pertenecen a la Red de Trastornos Adictivos de RETICS y en otros que manifestaron su interés en participar después de conocer la propuesta.

En su fase inicial, el proyecto cuenta con la participación de 10 centros así como de un biobanco que colecciona las muestras biológicas de los pacientes que se incluyen en la cohorte. Los centros participantes en el estudio, así como sus características se pueden ver en la Tabla 1 .

El inicio de reclutamiento de pacientes fue en Junio de 2013, momento en que el Comité Ético de Investigación Clínica (CEIC) del centro coordinador del estudio aprobó oficialmente el proyecto, si bien cada centro empezó el reclutamiento en función de la fecha de aprobación de su propio CEIC (Tabla 1). Los pacientes que se incluyen en la cohorte multicéntrica deben cumplir los siguientes criterios: ser mayor de 18 años, tener un diagnóstico de trastorno por uso de alcohol según criterios del DSMV(American Psychiatric Association, 2013) y firmar un consentimiento informado para la cesión de datos y muestras biológicas.

\section{Aspectos éticos}

Cada centro participante ha gestionado con su respectivo CEIC la aprobación del protocolo del estudio y el consentimiento informado que se administra a los pacientes en la primera visita. El consentimiento informado para la utilización de datos clínicos y muestras biológicas fue diseñado junto con miembros del Biobanco de la RTA situado en la Universidad Miguel Hernández, San Juan de Alicante y ha sido aprobado por cada uno de los CEIC de los centros participantes.

Además, el proyecto cuenta con el reconocimiento de la Agencia Española de Medicamentos y Productos Sanitarios como Estudio Observacional no Postautorización (No-EPA).

Los pacientes que firman el consentimiento informado lo hacen bajo la premisa de que la información que proporcionan es anónima y de que pueden revocar su consentimiento en cualquier momento, de conformidad con la Ley Española de Protección de Datos. En el consentimiento informado también se contempla la opción de que el paciente pueda ceder únicamente datos clínicos.

Tabla 1. Centros participantes en el estudio CohRTA, Programa Alcohol, Red de Trastornos Adictivos-RTA a Diciembre de 2015.

\begin{tabular}{|c|c|c|c|c|c|}
\hline Centro & Ciudad & Tipo de recurso asistencial & Perfil & Vinculación & Código CEIC \\
\hline $\begin{array}{l}\text { Hospital Univ. Germans } \\
\text { Trias i Pujol* }\end{array}$ & Badalona & Unidad de Adicciones & Medicina Interna & RETICS / PNSD & $\mathrm{PI}-13-031$ \\
\hline Hospital Clínic de Barcelona & Barcelona & Unidad de Alcohología & Psiquiatría & RETICS & $2013 / 8738$ \\
\hline Hospital Universitari de Bellvitge & L'Hospitalet de Llobregat & Unidad de Adicciones & Medicina Interna & PNSD & PR049/14 \\
\hline Hospital 12 de Octubre & Madrid & Servicio de Psiquiatría & Psiquiatría & RETICS & $15 / 065$ \\
\hline Universidad de Valladolid & Valladolid & $\begin{array}{l}\text { Alcohólicos Rehabilitados de } \\
\text { Valladolid (ARVA) }\end{array}$ & Atención primaria & RETICS & PI13-120 \\
\hline Hospital Universitari Son Espases & Palma de Mallorca & $\begin{array}{l}\text { Unidad de Problemas } \\
\text { Relacionados con el Alcohol } \\
\text { (UPRA) }\end{array}$ & Medicina Interna & PNSD & IB $2357 / 14 \mathrm{PI}$ \\
\hline Centro Delta & Badalona & $\begin{array}{l}\text { Centro Municipal de Atención a } \\
\text { las Drogodependencias }\end{array}$ & Atención primaria & PNSD & $\mathrm{PI}-13-031$ \\
\hline Universidad Miguel Hernández & San Juan de Alicante & Biobanco & & RETICS & \\
\hline
\end{tabular}

Nota. ${ }^{\star}$ Centro coordinador 


\section{Información registrada}

Se han diseñado dos cuestionarios estructurados, uno para la entrada del paciente en el estudio de cohorte y otro para las visitas de seguimiento.

En el cuestionario basal se incluyen variables sociodemográficas, antecedentes familiares, variables del consumo de alcohol y de sustancias de abuso y variables clínicas, analíticas y del tratamiento del trastorno.

Para evaluar la comorbilidad médica de los pacientes que entran en el estudio se utiliza el índice Cumulative Illnes Rating Scale-Substance Abuse (CIRS-SA) (Castillo et al., 2004). Se trata de una herramienta que analiza la presencia o ausencia de enfermedad en 13 órganos o sistemas: 1) Cardíaco, 2) Vascular, 3) Respiratorio, 4) Ojos, oídos, nariz, garganta y laringe, 5) Gastrointestinal alto, 6) Gastrointestinal bajo, 7) Hígado, 8) Renal, 9) Genitourinario, 10) Músculo-esquelético, 11) Neurológico, 12) Infecciones, endocrinología, metabólico, 13) Infección por VIH.

Además, cada órgano o sistema recibe una puntuación entre 0 y 4 según la severidad de la afectación: 0 , ausencia de afectación; 1, afectación leve (problema leve actual o antecedente de problema significativo); 2, afectación moderada (discapacidad moderada o morbilidad que requiere tratamiento de primera línea); 3, afectación severa (discapacidad severa/constante o problemas crónicos incontrolables); 4, afectación muy severa (extremadamente grave/requiere tratamiento inmediato/insuficiencia orgánica/deterioro severo de la función).

Para evaluar la comorbilidad psiquiátrica, el estudio CohRTA realiza un cribaje de depresión, psicosis, trastorno de atención e hipereactividad, estrés postraumático, ansiedad generalizada, pánico, fobia social y manía.

Un resumen detallado de estas variables se puede ver en la Tabla 2.

El cuestionario de seguimiento de los casos incluye datos sobre el consumo de alcohol posterior al primer tratamiento, además de incorporar la aparición de eventos clínicos y muerte.

Además, se ha previsto la opción de añadir al protocolo inicial del estudio una serie de módulos específicos para estudios adicionales; entre ellos, el cuestionario PRISM (Psychiatric Research Interview for Substance and Mental Disorder) ya se ha empezado utilizar en algunos centros. Otros como la valoración de la ideación suicida, uso de servicios de salud o calidad de vida están por determinar. Además, por ahora se ha implementado un modulo que contiene el cuestionario de salud general SF-12.

El cuestionario SF-12, consta de 12 preguntas que proporcionan una medida subjetiva del estado de salud. Evalúa ocho aspectos: estado físico, limitaciones por problemas físicos de salud, funcionamiento social, dolor corporal, salud mental, limitaciones por problemas personales o emocionales, vitalidad y salud general. Se trata de una versión reducida del cuestionario SF-36 que ha sido validada en
Tabla 2. Variables de estudio en los pacientes filiados en el estudio CohRTA, Programa Alcohol, Red de Trastornos Adictivos-RTA.

\begin{tabular}{|c|c|}
\hline Filiación & $\begin{array}{l}\text { Identificadores paciente } \\
\text { Fecha de nacimiento } \\
\text { Sexo } \\
\text { Fecha de inclusión } \\
\text { Recogida de muestras biológicas y fecha }\end{array}$ \\
\hline Sociodemográficas & $\begin{array}{l}\text { Estado civil } \\
\text { Situación socioeconómica y laboral } \\
\text { Nivel de estudios }\end{array}$ \\
\hline Diagnóstico & $\begin{array}{l}\text { Severidad del trastorno por uso } \\
\text { de alcohol según DSMV }\end{array}$ \\
\hline Consumo alcohol & $\begin{array}{l}\text { Historia de consumo } \\
\text { Patrón consumo actual* (cantidad y frecuencia) } \\
\text { Tiempo de abstinencia } \\
\text { Intoxicaciones etílicas } \\
\text { Antecedentes familiares }\end{array}$ \\
\hline $\begin{array}{l}\text { Consumo otras } \\
\text { sustancias }\end{array}$ & $\begin{array}{l}\text { Tabaco (historia de consumo, } \\
\text { patrón consumo actual) } \\
\text { Cocaína (historia de consumo, } \\
\text { patrón consumo actual) } \\
\text { Cannabis (patrón consumo actual) } \\
\text { Anfetaminas (patrón consumo actual) } \\
\text { Alucinógenos (patrón consumo actual) } \\
\text { Opiáceos (patrón consumo actual) } \\
\text { Inhalantes (patrón consumo actual) } \\
\text { Drogas emergentes (patrón consumo actual) } \\
\text { Drogas por vía intravenosa (historia de consumo, } \\
\text { consumo actual) }\end{array}$ \\
\hline Analítica general & $\begin{array}{l}\text { Hemograma ( } 10 \text { parámetros) } \\
\text { Bioquímica ( } 28 \text { parámetros) } \\
\text { Estudio de anemias ( } 8 \text { parámetros) } \\
\text { Serologías víricas ( } 10 \text { parámetros) } \\
\text { Drogas de abuso en orina (opiáceos, cocaína, } \\
\text { cannabis, anfetaminas) }\end{array}$ \\
\hline Comorbilidad & $\begin{array}{l}\text { Orgánica según índice CIRS-SA** } \\
\text { Psiquiátrica según cribaje distintas patologías }\end{array}$ \\
\hline Tratamiento & $\begin{array}{l}\text { Farmacológico (fármaco, fecha inicio y final, } \\
\text { dosis prescrita) } \\
\text { Intervenciones terapéuticas no farmacológicas } \\
\text { (tipo y fecha) }\end{array}$ \\
\hline
\end{tabular}

Nota. *Se considera consumo actual el realizado los últimos 30 días; ${ }^{\star *}$ Cumulative Illnes Rating Scale-Substance Abuse.

distintas poblaciones y países y que es sensible a cambios terapéuticos (Gandek et al., 1998; Salyers, Bosworth, Swanson, Lamb-Pagone, y Osher, 2000).

\section{Proceso de recogida de datos}

Cada centro participante tiene asignada una persona encargada de la recogida de datos de los pacientes en estudio. El almacenaje de la información y las actualizaciones periódicas se hacen mediante una plataforma diseñada para dicho fin (Coresfot Clínico, www.coresoft.es ) por profesionales dedicados al desarrollo, mantenimiento y soporte de registros clínicos online que cumple con la Ley Orgánica de Protección de Datos, así como con las leyes vigentes sobre seguridad y confidencialidad. La implementación del aplicativo para la introducción de datos se ha 
hecho en dos fases: una primera fase (septiembre 2014) en la que estaban disponibles los módulos de información referentes a las variables sociodemográficas y de consumo de drogas y en la cual únicamente tenían acceso los grupos pertenecientes a la RTA y una segunda fase (septiembre 2015) en la que se implementaron los módulos clínicos y en la que se abrió el acceso a la plataforma a aquellos grupos clínicos incluidos en la ayuda adicional del PNSD.

\section{Biobanco}

Los centros participantes en el estudio CohRTA disponen de un biobanco de referencia para la recepción y almacenamiento de muestras biológicas. Cada muestra deber estar identificada con el código del paciente, de forma que permita su conexión con la base de datos clínicos. Para la recogida de muestras biológicas se extraen 10 mL de sangre que será procesada para obtener ADN.

El biobanco del proyecto CohRTA está situado en el Instituto de Neurociencias de la Universidad Miguel Hernández, en San Juan de Alicante. El biobanco dispone de un comité científico formado por el coordinador de la RTA, un responsable local e investigadores de la red. El sistema de gestión del biobanco cumple con la normativa internacional UNE-EN-ISO 9001:2008 ( $\mathrm{N}^{\circ}$ Registro ER0614/2010).

Según la normativa vigente, las muestras biológicas pueden cederse a proyectos de investigación vinculados a la RTA y relacionados con el trastorno por uso de alcohol, previa autorización de un Comité de Ética.

\section{Criterios de autoría}

El proyecto CohRTA dispone de un documento con los criterios que se seguirán para la autoría científica de los trabajos que se deriven del proyecto. Estos criterios se basan en establecer un orden de autorías en la cabecera de los artículos científicos y comunicaciones a congresos y en determinar el contenido del Apéndice que recoge los distintos participantes y centros de investigación. Se establecen criterios de autorías distintos en función del grado de participación, utilización de muestras y/o datos clínicos o de si los trabajos han sido desarrollados por miembros del proyecto CohRTA o por otros investigadores preclínicos de la RTA que pudieran utilizar muestras biológicas de pacientes incluidos en CohRTA.

\section{Análisis de datos}

Se extrajeron del aplicativo las variables sociodemográficas y de consumo de alcohol y otras drogas de todos los pacientes registrados entre junio 2013 y noviembre 2015. Se realizó un análisis descriptivo de los datos en el cual las variables continuas se describieron mediante la mediana y el rango intercuartílico (RIQ) y las variables categóricas mediante las frecuencias relativas. $\mathrm{El}$ análisis estadístico fue realizado con SPSS 15.0 (SPSS, Chicago, IL, USA).

\section{Resultados}

Entre junio de 2013 y noviembre de 2015 se filiaron 344 pacientes, $264(76.7 \%)$ hombres, con una mediana de edad de 50 años (RIQ:43-55 años). La edad de inicio de consumo de alcohol fue 15 años (RIQ:14-18 años) y un $61 \%$ referían antecedente familiar de trastorno por uso de alcohol. Durante los 30 días previos al inicio de tratamiento, los pacientes bebían una mediana de $12.5 \mathrm{UBE} /$ día (RIQ:7.1-20 UBE/día), un 72\% eran fumadores de tabaco y un $29.7 \%$ consumían cocaína. Los datos sociodemográficos y los relacionados con la historia de consumo de alcohol y otras sustancias se muestran en la Tabla 3 .

De los pacientes incluidos en el proyecto CohRTA se dispone actualmente de 76 muestras para el estudio del ADN. El hecho de que el estudio esté asociado a un repositorio de muestras biológicas dará lugar a un número de proyectos en los que grupos preclínicos con experiencia en modelos animales trasladen sus hipótesis a la investigación en humanos.

Dado el potencial de información clínica y biológica que se puede recoger mediante este estudio, puede servir de plataforma para la formación de nuevos investigadores clínicos en España y la elaboración de tesis doctorales. La incorporación de distintos centros y la implicación de grupos clínicos asistenciales en entornos científicos pueden potenciar la investigación al disponer de métodos adecuados para responder a retos del diagnóstico, pronóstico y tratamiento de la enfermedad. Además, el sistema nacional de salud y la política científica de RETICS apoyan este tipo de proyectos basados en estructuras nacionales de investigación y centrados en el paciente. Programas de formación en investigación del ISCIII como Rio Hortega o Juan Rodés están orientados a mejorar las habilidades investigadoras de jóvenes facultativos.

En definitiva, con una cohorte de pacientes bien establecida se espera aumentar la cantidad y calidad científica en relación a las complicaciones del trastorno por uso de alcohol y sus consecuencias clínicas y sociales en España.

\section{Discusión}

La demanda de tratamiento del trastorno por uso de alcohol en España ha aumentado. El perfil de los pacientes que solicitan tratamiento del trastorno es el de adultos de mediana edad, mayoritariamente hombres y que pueden ser consumidores de cocaína y de cannabis. Este perfil es similar al descrito en pacientes con trastorno por uso de alcohol o consumo de alcohol de riesgo a los que se les realiza una intervención breve en salas de urgencias, atención primaria u hospitalizados por otras causas (Heather, 2014; Mdege et al., 2013; Nilsen et al., 2008). Los resultados que se muestran en este estudio revelan la severidad del trastorno en aquellos que solicitan tratamiento por primera vez y confirman que éste se produce, en promedio, casi 30 años 
Tabla 3. Características sociodemográficas y del consumo de alcohol y otras sustancias en 344 pacientes con trastorno por uso de alcohol que solicitan tratamiento por primera vez. Programa Alcohol, Red de Trastornos Adictivos-RTA.

\begin{tabular}{lr}
\hline & $\begin{array}{r}\mathbf{N = 3 4 4} \\
\mathbf{n}(\%)\end{array}$ \\
\hline Sociodemográficas & $264(76.7)$ \\
\hline Hombres & $50[43-55]$ \\
\hline Edad, mediana [RIQ] & $324(94.2)$ \\
\hline Españoles & $89(26.6)$ \\
\hline Estado civil (n=335) & $138(41.2)$ \\
Soltero/a & $11(3.3)$ \\
Casado/a - Pareja de Hecho & $97(28.9)$ \\
Viudo/a & $152(45.1)$ \\
Separado/a - Divorciado/a & $104(30.9)$ \\
\hline Situación laboral (n=337) & $69(20.5)$ \\
Trabajando & $12(3.6)$ \\
Parado & \\
Incapacidad permanente / pensionista & \\
Otras situaciones & \\
\hline Consumo de alcohol y otras drogas &
\end{tabular}

Severidad del trastorno por uso de alcohol

según DSM-V $(n=340)$

$2-5$

$62(18.2)$

6-8

$169(49.7)$

$9-11$

$109(32.1)$

Edad inicio consumo alcohol, mediana [RIQ] 15 [14-18]

Edad inicio del consumo regular alcohol, mediana [RIQ] 22 [18-30]

UBE / día, últimos 30 días, mediana [RIQ] 12.5 [7.1-20]

Tiempo total abstinencia al alcohol (años), mediana [RIQ] 1 [0-3]

Antecedente familiar de trastorno

por uso de alcohol $(n=334)$

$203(60.8)$

Número de intoxicaciones etílicas con atención médica

a lo largo de la vida $(n=321)$

$\begin{array}{ll}\text { Ninguna } & 147(45.8) \\ 1-5 & 162(50.5)\end{array}$

$162(50.5)$

$>5$

$12(3.7)$

Tabaco $(n=341)$

Si

No

$245(71.8)$

$62(18.2)$

Exfumador

$34(10.0)$

Consumo en los últimos 30 días de:

Cocaína

$33(29.7)$

Cannabis/marihuana

Anfetaminas

Tranquilizantes o benzodiacepinas sin prescripción médica

$78(22.9)$

$9(2.6)$

$20(5.9)$

Opiáceos sin prescripción médica

4 (1.2)

Consumo de drogas por vía parenteral alguna vez en la vida

$12(3.5)$

después de iniciar el consumo de alcohol. Una revisión sistemática reciente del trastorno indica que el primer episodio de tratamiento es tardío y se produce cuando está clínicamente establecido (Connor, Haber y Hall, 2016). Por otro lado, observamos que dos de cada tres pacientes tenían antecedentes familiares de trastorno por uso de alcohol que, de hecho, es uno de los factores de riesgo ya descritos para esta enfermedad (Connor et al., 2016).

Debido a la escasez de estudios multicéntricos españoles en esta patología, el proyecto de investigación aquí presen- tado permitirá conocer la dimensión clínica del problema, su tratamiento y en definitiva actualizará el pronóstico de la enfermedad. Además, el estudio aportará conocimiento en el abuso de alcohol en la mujer y sus secuelas a largo plazo, hasta ahora no bien delimitadas; las mujeres con trastorno por uso de alcohol representan sólo un $20 \%$ del total y se requiere de un amplio reclutamiento de casos para obtener una muestra representativa. Por otra parte, los resultados obtenidos en este proyecto podrán ser de utilidad para plantear nuevas estrategias diagnósticas del trastorno.

La capacidad de investigación y de transferencia del conocimiento es clave en proyectos multicéntricos. El trabajo en red es una oportunidad para dar proyección a la investigación centrada en el paciente. Las ventajas de la integración de grupos clínicos y básicos con intereses afines son innumerables y el proyecto CohRTA puede ser una plataforma adecuada para adaptarse a la investigación del futuro.

Los estudios de cohorte han supuesto un cambio radical en el conocimiento de algunas enfermedades. Solo a modo de ejemplo, buena parte del conocimiento actual en el riesgo de desarrollar enfermedades cardiovasculares se debe al estudio Framingham (EE.UU), una cohorte prospectiva que se empezó a reclutar en 1948 para establecer los hoy en día más que reconocidos factores de riesgo cardiovascular (McKee, Castelli, McNamara, y Kannel, 1971). Años después el modelo de investigación longitudinal en esta enfermedad se implantó en nuestra sociedad (Nascetti et al., 2001) como también ha ocurrido en otras como el VIH/Sida (Sobrino-Vegas et al., 2011) y la enfermedad tromboembólica (Nieto y Monreal, 2004); todas ellas han incrementado de forma notable el conocimiento a través de una producción científica excelente en sus respectivas áreas de conocimiento.

En el caso del trastorno por uso de alcohol y de la adicción a las drogas, son varios los estudios de cohorte que se han desarrollado en nuestro entorno. Cabe citar, por ejemplo, la cohorte de 850 pacientes que solicitaron tratamiento por abuso de alcohol a finales de los años ochenta (Gual, Lligona, y Colom, 1999; Gual, Lligoña, Costa, Segura, y Colom, 2004), la cohorte Itinere que incluye pacientes usuarios de heroína y cocaína (Pulido et al., 2009) o la de cerca de 6.000 pacientes ingresados en unidades hospitalarias de desintoxicación de Barcelona y área metropolitana, 1.200 de los cuales por dependencia del alcohol (Rivas et al., 2013; Sanvisens et al., 2014).

El proyecto CohRTA está específicamente diseñado para analizar pacientes con trastorno por uso de alcohol desde el momento que solicitan tratamiento del trastorno por primera vez; el estudio está anidado en centros de primera línea asistencial, es rico en datos clínicos y biológicos, está orientado al paciente y se centra en conocer el impacto del alcoholismo a medio y largo plazo. No obstante, este tipo de estudios presenta varias limitaciones la principal de las cuales deriva de su carácter longitudinal. Los estudios 
longitudinales son costosos, requieren especial dedicación para el seguimiento de todos los casos durante un largo periodo de tiempo y personal cualificado para la actualización de datos y análisis estadístico. Por otro lado, el carácter multicéntrico de este estudio puede tener un cierto sesgo interobservador para determinadas variables que requieren de interpretación (ej. comorbilidad clínica y psiquiátrica) si bien este aspecto se ha procurado minimizar mediante la elaboración de guías ampliamente detalladas para la recogida de datos.

Sin embargo, el nivel científico de los grupos participantes en CohRTA ya sea en el tratamiento del trastorno (Gual y Miquel, 2015; López-Pelayo et al., 2014), neuroinflamación y daño cerebral (Montesinos et al., 2015; Pascual, Baliño, Aragón, y Guerri, 2015), inmunidad y genética asociada a la hepatopatía alcohólica (Chamorro et al., 2014; Novo-Veleiro et al., 2014), comorbilidad y mortalidad (Rivas et al., 2013; Sanvisens et al., 2014) o dimensión del problema desde la Salud Pública (Bosque-Prous et al., 2014; Villalbí, Bosque-Prous, Gili-Miner, Espelt, y Brugal, 2014) garantizan la viabilidad del proyecto.

\section{Agradecimientos}

El Proyecto CohRTA está parcialmente financiado por el Ministerio de Economía y Competitividad, Instituto de Salud Carlos III (RETICS RD12/0028 y RD16/0017), Fondo Europeo de Desarrollo Regional (FEDER) y por el Plan nacional Sobre Drogas, Ministerio de Sanidad, Política Social e Igualdad. (PNSD 2014|042, PNSD 2015| 027 y PNSD 2015|054). Esther Papaseit está adscrita al programa Joan Rodés del Instituto de Salud Carlos III (JR16/00020). Fulbright Scholar Program - Ministerio de Educación (PRX16/00147).

\section{Apéndice}

Red de Trastornos Adictivos-RTA, Coordinación del Programa Alcohol: Fernando Rodríguez de Fonseca, Instituto de Investigación Biomédica de Málaga, Málaga.

Proyecto CohRTA, Comité ejecutivo: Antoni Gual, Francisco Javier Laso, Marta Torrens, Fernando Rodríguez de Fonseca, Robert Muga.

Proyecto CohRTA, Centro Coordinador: Servicio de Medicina Interna, Hospital Universitari Germans Trias i Pujol, Badalona.

Proyecto CohRTA, Centros participantes e investigadores del estudio a 31 de Diciembre de 2015: Hospital Universitari Germans Trias i Pujol, Badalona: Paola Zuluaga, Daniel Fuster, Jordi Tor, Robert Muga, Magí Farré, Arantza Sanvisens, Esther Papaseit; Hospital Clínic, Barcelona: Antoni Gual, Laia de Miquel; Parc de Salut Mar, Barcelona: Marta Torrens, Francina Fonseca, Gabriel Vallecillo, Alvaro Palma; Hospital Universitari de Bellvitge, L'Hospitalet de
Llobregat: Ferran Bolao; Hospital Clínico Universitario, Salamanca: Francisco Javier Laso, Miguel Marcos; Hospital 12 de Octubre, Madrid: Gabriel Rubio, Núria García-Marchena; Hospital Clínico Universitario de Valladolid-Alcohólicos Rehabilitados de Valladolid (ARVA), Valladolid: Javier Álvarez, Trinidad Gómez-Talegón; Hospital Lucus Augusti, Lugo: Rafael Monte; Hospital Universitario Son Espases, Palma de Mallorca: Antoni Short, Catalina Moranta, Rafael Blanes; Centro de Atención a las Drogodependencias-Centro Delta, Badalona: Eva Faure, Néstor Espinach, Inmaculada Rivas.

Biobanco: Luis Navarro, Carmen de Felipe; Instituto de Neurociencias, Universidad Miguel Hernández, San Juan de Alicante.

\section{Conflictos de interés}

No existen conflictos de interés.

\section{Referencias}

American Psychiatric Association. (2013). Diagnostic and statistical manual of mental disorders (5th ed.). Wasington, DC: American Psychiatric Association.

Bagnardi, V., Blangiardo, M., La Vecchia, C. y Corrao, G. (2001). Alcohol consumption and the risk of cancer: a meta-analysis. Alcohol Research E Health, 25, 263-270.

Bosque-Prous, M., Espelt, A., Guitart, A. M., Bartroli, M., Villalbí, J. R. y Brugal, M. T. (2014). Association between stricter alcohol advertising regulations and lower hazardous drinking across European countries. Addiction, 109, 1634-1643. doi: 10.1111/add.12562.

Castillo, C., Bulbena, A., Serras, E., Torrens, M., López-Colomés, J. L., Martínez, M. A. y Politinska, B. (2004). Medical assessment in drug addicts: reliability and validity of the Cumulative Illness Rating Scale (Substance Abuse version). European Addiction Research, 10, 112-117. doi: 10.1159/000077699.

Chamorro, A. J., Torres, J. L., Mirón-Canelo, J. A., González-Sarmiento, R., Laso, F. J. y Marcos, M. (2014). Systematic review with meta-analysis: the $1148 \mathrm{M}$ variant of patatin-like phospholipase domain-containing 3 gene (PNPLA3) is significantly associated with alcoholic liver cirrhosis. Alimentary Pharmacology $\mathcal{E}$ Therapeutics, 40, 571-581.doi: 10.1111/apt.12890.

Connor, J. P., Haber, P. S. y Hall, W. D. (2016). Alcohol use disorders. Lancet, 387, 988-998. doi: 10.1016/S01406736(15)00122-1

Fuster, D., Sanvisens, A., Bolao, F., Serra, I., Rivas, I., Tor, J. y Muga, R. (2015). Impact of hepatitis C virus infection on the risk of death of alcohol-dependent patients. Journal of Viral Hepatitis, 22, 18-24.doi: 10.1111/jvh.12290.

Gandek, B., Ware, J. E., Aaronson, N. K., Apolone, G., Bjorner, J. B., Brazier, J. E.,... Sullivan, M. (1998) . Cross-vali- 
dation of item selection and scoring for the SF-12 Health Survey in nine countries: results from the IQOLA Project. International Quality of Life Assessment. Journal of Clinical Epidemiology, 51, 1171-1178.

Gossop, M., Manning, V. y Ridge, G. (2006). Concurrent use and order of use of cocaine and alcohol: behavioural differences between users of crack cocaine and cocaine powder. Addiction, 101, 1292-1298.doi: 10.1111/j.13600443.2006.01497.x.

Gual, A., Lligona, A. y Colom, J. (1999). Five-year outcome in alcohol dependence. A naturalistic study of 850 patients in Catalonia. Alcohol and Alcoholism, 34, 183-192.

Gual, A., Lligoña, A., Costa, S., Segura, L. y Colom, J. (2004).Tratamiento del alcoholismo y su impacto a largo plazo. Resultados a 10 años de un estudio longitudinal prospectivo de 850 pacientes. Medicina Clínica (Barcelona), 123, 364-369.

Gual, A. y Miquel, L. (2015). Nuevas perspectivas para el tratamiento del alcoholismo. Medicina Clínica (Barcelona), 144, 24-25. doi: 10.1016/j.medcli.2014.07.020.

Heather, N. (2014). The two forms of alcohol brief intervention: an uneasy coalition. Addiction, 109, 1059-1060. doi: 10.1111/add.12443.

López-Pelayo, H., Wallace, P., Segura, L., Miquel, L., Díaz, E., Teixidó, L.,... Gual, A. (2014). A randomised controlled non-inferiority trial of primary care-based facilitated access to an alcohol reduction website (EFAR Spain): the study protocol. BMJ Open, 4, e007130.doi: 10.1136/bmjopen-2014-007130.

McKee, P. A., Castelli, W. P., McNamara, P. M. y Kannel, W. B. (1971). The natural history of congestive heart failure: the Framingham study. The New England Journal of Medicine, 285, 1441-1446. doi: 10.1056/ NEJM197112232852601.

Mdege, N. D., Fayter, D., Watson, J. M., Stirk, L., Sowden, A. y Godfrey C. (2013). Interventions for reducing alcohol consumption among general hospital inpatient heavy alcohol users: a systematic review. Drug and Alcohol Dependence, 131, 1-22. doi: 10.1016/j.drugalcdep.2013.01.023.

Montesinos, J., Pascual, M., Pla, A., Maldonado, C., Rodríguez-Arias, M., Miñarro, J. y Guerri, C. (2015). TLR4 elimination prevents synaptic and myelin alterations and long-term cognitive dysfunctions in adolescent mice with intermittent ethanol treatment. Brain, Behavior and Immunity, 45, 233-244. doi: 10.1016/j.bbi.2014.11.015.

Naimi, T. S., Brewer, R. D., Mokdad, A., Denny, C., Serdula, M. K. y Marks, J. S. (2003). Binge drinking among US adults. JAMA, 289, 70-75.

Nascetti, S., Elosua, R., Pena, A., Covas, M. I., Senti, M. y Marrugat, J. (2001). Variables associated with fibrinogen in a population-based study: interaction between smoking and age on fibrinogen concentration. European Journal of Epidemiology, 17, 953-958.
Nieto, J. A. y Monreal, M. (2004). Recurrent venous thromboembolism in men and women. The New England Journal of Medicine, 351, 2015-2018.

Nilsen, P., Baird, J., Mello, M. J., Nirenberg, T., Woolard, R., Bendtsen, P. y Longabaugh, R. (2008). A systematic review of emergency care brief alcohol interventions for injury patients. Journal of Substance Abuse Treatment, 35, 184-201.doi:10.1016/j.jsat.2007.09.008.

Novo-Veleiro, I., González-Sarmiento, R., Cieza-Borrella, C., Pastor, I., Laso, F. J. y Marcos, M. (2014). A genetic variant in the microRNA-146a gene is associated with susceptibility to alcohol use disorders. European Psychiatry, 29, 288-292.doi: 10.1016/j.eurpsy.2014.02.002.

Observatorio Español sobre Drogas, Delegación del Gobierno para el Plan Nacional sobre Drogas. (2011). Informe 2011. Situación y tendencias de los problemas de drogas en España. Madrid: Ministerio de Sanidad, Política Social e Igualdad.

Pascual, M., Baliño, P., Aragón, C. M. G. y Guerri, C. (2015). Cytokines and chemokines as biomarkers of ethanol-induced neuroinflammation and anxiety-related behavior: role of TLR4 and TLR2. Neuropharmacology, 89, 352-359. doi: 10.1016/j.neuropharm.2014.10.014.

Pennings, E. J. M., Leccese, A. P. y de Wolff, F. A. (2002). Effects of concurrent use of alcohol and cocaine. Addiction, 97, 773-783.

Pons, J. M. V., Rodés, J., Andreu, A. y Arenas, J. (2013). La olvidada investigación clínica. Medicina Clínica (Barcelona), 140, 325-331.doi: 10.1016/j.medcli.2012.10.011.

Pulido, J., Brugal, M. T., de la Fuente, L., Ballesta, R., Barrio, G., Bravo, M. J.,... Fernández, F. (2009). Metodología de reclutamiento y características de una cohorte de jóvenes consumidores habituales de cocaína de tres ciudades españolas. Gaceta Sanitaria, 23, 200-207.doi: 10.1016/j.gaceta.2008.05.003.

Rivas, I., Sanvisens, A., Bolao, F., Fuster, D., Tor, J., Pujol, R.,... Muga, R. (2013). Impact of medical comorbidity and risk of death in 680 patients with alcohol use disorders. Alcoholism, Clinical and Experimental Research, 37 Suppl 1, E221-E227.doi: 10.1111/j.15300277.2012.01861.x.

Roerecke, M. y Rehm, J. (2013). Alcohol use disorders and mortality: a systematic review and meta-analysis. Addiction, 108, 1562-1578.doi: 10.1111/add.12231.

Salyers, M. P., Bosworth, H. B., Swanson, J. W., Lamb-Pagone, J. y Osher, F. C. (2000). Reliability and validity of the SF-12 health survey among people with severe mental illness. Medical Care, 38, 1141-1150.

Sanvisens, A., Vallecillo, G., Bolao, F., Rivas, I., Fonseca, F., Fuster, D.,... Muga, R. (2014). Temporal trends in the survival of drug and alcohol abusers according to the primary drug of admission to treatment in Spain. Drug and Alcohol Dependence, 136, 115-120. doi: 10.1016/j.drugalcdep.2013.12.022. 
Sobrino-Vegas, P., Gutiérrez, F., Berenguer, J., Labarga, P., García, F., Alejos-Ferreras, B.,... del Amo, J. (2011). La cohorte de la red española de investigación en Sida y su biobanco: organización, principales resultados y pérdidas de seguimiento . Enfermedades Infecciosas y Microbiología Clínica, 29, 645-653.doi: 10.1016/j.eimc.2011.06.002.

Thomas, D. L., Astemborski, J., Rai, R. M., Anania, F. A., Schaeffer, M., Galai, N.,... Vlahov, D. (2000). The natural history of hepatitis $\mathrm{C}$ virus infection: host, viral, and environmental factors. JAMA, 284, 450-456.

Villalbí, J. R., Bosque-Prous, M., Gili-Miner, M., Espelt, A. y Brugal, M. T. (2014). Políticas para prevenir los daños causados por el alcohol. Revista Española de Salud Pública, 88, 515-528.doi: 10.4321/S1135-57272014000400006.

World Health Organization. (2010). Global Status Report on Alcohol. Ginebra: World Health Organization. 